\title{
Autistic traits and gender modulate emotion changes before and during the COVID-19 pandemic
}

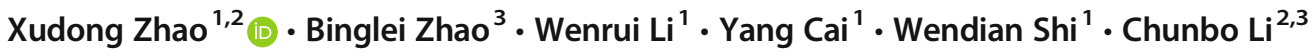 \\ Accepted: 29 July 2021 / Published online: 7 August 2021 \\ (C) The Author(s), under exclusive licence to Springer Science+Business Media, LLC, part of Springer Nature 2021
}

\begin{abstract}
Individuals with autism spectrum disorder (ASD) show an enhanced response to stressors, and gender plays an important role in stress response. Thus, autistic traits (ATs) in the general population and gender may regulate the emotion changes before and during the COVID-19 pandemic. In the present study, participants were divided into higher and lower ATs groups. The generalized linear models were used to estimate the effects of the independent variables (e.g. the COVID-19 pandemic status (before, during), gender (male, female), and AT groups (higher ATs, lower ATs) and their interactions on emotions measured by the Positive and Negative Affect scales. The results showed that the COVID-19 pandemic reduced positive emotions and increased fear and anger. Furthermore, compared with the status before the COVID-19 pandemic, individuals with higher ATs and females experienced stronger anger and fear than individuals with lower ATs and males during the pandemic. The present study revealed the emotional impacts of the COVID-19 pandemic and greater emotional susceptibility to the pandemic among individuals with higher ATs and females. Our findings provide prospective evidence for understanding the ASD/ATsrelated enhanced response to pathogen threat-related stressors and have implications for COVID-19 crisis interventions.
\end{abstract}

Keywords Autism Spectrum disorders $\cdot$ Autistic traits $\cdot$ COVID-19 $\cdot$ Gender $\cdot$ Positive emotions $\cdot$ Negative emotions

\section{Introduction}

Autism spectrum disorder (ASD) is a set of neurodevelopmental conditions, characterized by impaired social functions and unusually restricted behaviours and interests (Baron-Cohen et al., 2001). Autistic traits (ATs) are considered the primary symptoms of ASD and are assumed to be distributed continuously amongst the general population (Zhao et al., 2019). Quantifying the differences in cognitive-emotional function associated with ATs can provide an indispensable alternative for the understanding of ASD symptoms (Haruvi-Lamdan et al., 2019).

Wendian Shi

swd_nx@163.com

$\triangle$ Chunbo Li

licb@smhc.org.cn

1 Department of Psychology, School of Education, Shanghai Normal University, Shanghai, China

2 Shanghai Key Laboratory of Psychotic Disorders, Shanghai Mental Health Center, Shanghai Jiao Tong University School of Medicine, Shanghai, China

3 Institute of Psychology and Behavioral Science, Shanghai Jiao Tong University, Shanghai, China
Some endogenous characteristics associated with ASD, such as executive dysfunction, sensory hypersensitivity, poor emotional regulation, and difficulties in socialization and communication, are likely to predispose individuals with ASD to be especially vulnerable to stressors (BishopFitzpatrick et al., 2015). For instance, individuals diagnosed with ASD reported significantly higher subjective stress regarding general life and experimental stressors (BishopFitzpatrick et al., 2015; Bishop-Fitzpatrick et al., 2017), as well as exhibited increased peripheral physiological responses to stressors, marked with enhanced cortisol release and increased heart rate, as compared to the typical development (TD) controls (Spratt et al., 2012; Taylor \& Corbett, 2014; van Oort et al., 2020). Furthermore, these individuals do not show decreased default mode network connectivity nor increased frontoparietal network connectivity in response to stressors compared with TD controls (van Oort et al., 2020). These findings indicated that individuals with ASD may fail to transfer effectively attention resources from their internal emotional state to the external world, and that they fail to fully invoke emotional regulation and executive functions to support adaptive coping when facing stressors (van Oort et al., 2020). Evidence on relation between ASD/ATs and increased posttraumatic stress disorder symptoms (PTSS), especially in 
the hyper-arousal and negative emotion clusters (HaruviLamdan et al., 2020; Haruvi-Lamdan et al., 2019; Nirit et al., 2018), has further consolidated the atypical stress mechanism associated with ASD. In sum, all these findings suggest that individuals with ASD or higher ATs may have an enhanced response (e.g. emotion-related) to or are more susceptible to the negative consequences of stressors.

Nevertheless, previous studies have assessed selfperceived stress in individuals with ASD as a general sense of stress, such as stress measured by the Perceived Stress Scale (Bishop-Fitzpatrick et al., 2017; Hirvikoski \& Blomqvist, 2015), rather than based on specific stressful events. The stress response of individuals with ASD or higher ATs is more likely to be regulated by the category of stressors (Taylor \& Corbett, 2014), and as such, the response characteristics of these individuals in different stress situations need to be further elucidated. In the study of the relation between ASD/ATs and PTSS, though the measured PTSS is based on a specific stressor, type of stressor has a great deal of within-group variation, because participants' responses are based on the most traumatic events they have individually chosen from the list of stressful and traumatic life events (Haruvi-Lamdan et al., 2020; Haruvi-Lamdan et al., 2019), which means that the stressor types are almost different for each participant. Furthermore, in contrast to TD controls, individuals with ASD tend to perceive social stressors as their most traumatic event (Haruvi-Lamdan et al., 2020; Haruvi-Lamdan et al., 2019). This overall bias in event types between groups is likely to limit the inter-group comparability of the measured PTSS. Therefore, comparing the differences of the negative output of the similar stressors among groups (e.g. ASD vs. TD or higher ATs vs. lower ATs) is helpful to shed light on the atypical stress mechanism of autism spectrum.

The public has been exposed to a considerable stressor that is the COVID-19 pandemic (Wang et al., 2021). The COVID19 pandemic related stress is an integration of fear to virus infection and the incidental negative psychological and practical effects of measures to prevent the spread of the virus (Valle et al., 2020). Reduced positive emotions and increased negative emotions, especially fear and anger, are one of the main manifestations of COVID-19 pandemic stress (Huang et al., 2020; Li et al., 2020; Moron \& Biolik-Moron, 2021; Wang et al., 2021). Identifying the susceptibility population of the emotional impact of current pandemic is highly significant for the public policy-making and crisis intervention (Zhao et al., 2021). Individuals with higher ATs or ASD are one of the candidates.

ASD is associated with an increased risk of pathogen infection or disease related to immune system malfunctions (Masi et al., 2015; Saresella et al., 2009). According to the risk-as-feelings model (Tanu \& Kakkar, 2019), these cognitive and emotional experiences associated with pathogen infection may lead to individuals with ASD or higher ATs having an increased awareness of pathogen threat and fear to avoid the recurrence of similar disease experiences. In addition, some endogenous or co-occurring characteristics associated with ASD or higher ATs, such as lower tolerance of uncertainty and unexpected change (Kildahl et al., 2019), may make more challenging for these individuals in dealing with a series of negative consequences caused by the pandemic (Cassidy et al., 2020), such as the increased uncertainty and changes in routines (Valle et al., 2020), so as to experience more non-adaptive emotions. However, the association between ASD/ATs and the possible enhanced response to COVID-19 pandemic has not been identified. Thus, it is an opportunity to dig out how ATs regulate emotion-related stress response, from the perspective of complex stressor in which the COVID-19 pandemic includes both pathogen threat and widespread incidental negative consequences: almost all people are more or less affected by a similar stressor.

Gender has also been suggested to play an important role in stress response. Females are more susceptible to experience PTSS than males owing to genetic and biological risk factors (Farhood et al., 2018; Haruvi-Lamdan et al., 2020), and tend to show more negative emotions, such as anger and fear after natural disasters (Shavit et al., 2013) and during the current COVID-19 pandemic (Huang et al., 2020; Isabelle \& Kleinberg, 2020). The association between ASD/ATs and trauma exposure and PTSS mainly brought by social stressors may be stronger in females than in males (Haruvi-Lamdan et al., 2020; Haruvi-Lamdan et al., 2019). However, owing to the significant imbalance in the proportion of male and female in clinical studies, whether this gender inequality association can also be applied in the response to the COVID-19 pandemic needs to be further verified based on large-sample studies with a relatively balanced gender ratio.

Taken together, autism spectrum is likely to be endogenously associated with impaired stress mechanism. However, there is a lack of description of whether individuals with higher ATs exhibit distinct responses to COVID-19 pandemic and of the role of gender. Based on a cross-sectional design, which has been used to examine the changes in dreams and self-concept before and during COVID-19/COVID-19 lockdown (Barrett, 2020; González-Valero et al., 2020), the present study first compared the changes in positive and negative emotions measured by the Positive and Negative Affect Schedule (PANAS) (Watson et al., 1988), as well as anger (Irritable) and fear (Scared and Afraid) in the negative emotions subscale that seem to be particularly sensitive to the emotional impact of COVID-19 pandemic ( $\mathrm{Li}$ et al., 2020; Moron \& Biolik-Moron, 2021), before and during the COVID-19 pandemic. Moreover, we further explored the moderating effect of ATs and gender on the emotional impacts of COVID-19 pandemic. We hypothesized that the COVID19 pandemic reduced positive emotions but increased negative emotions, especially fear and anger. We further 
predicated that females and individuals with higher ATs, especially females with higher ATs, were more susceptible to the emotional effects of COVID-19 pandemic.

\section{Methods}

\section{Participants}

Convenience sampling was used to select participants (college students) before and during the COVID-19 pandemic. All of the participants before the outbreak were recruited offline in Shanghai, China, whereas the participants during the COVID19 pandemic were recruited online from universities located in various regions of China (see Table 2 for details). A total of 1374 university students were recruited before $(n=580)$ and during $(n=794)$ the COVID-19 outbreak. The questionnaires with incomplete answers or that showed items being consistently selected for the highest or lowest score were eliminated. In total, 558 (304 females) pre- and 721 (385 females) duringCOVID-19 valid participants were included for data analysis.

All the participants gave written or electronic informed consent to participate in the study after the nature of the study had been explained to them.. All of them reported no history of psychiatric, neurological, or other serious medical illness, and no direct relatives with an ASD diagnosis.

To investigate the regulatory effect of ATs on the emotional impacts of COVID-19, we used the quartile (Q3 and Q1) scores in the Autism Spectrum Quotient (AQ) to group the AT groups: participants who scored higher than Q3 and lower than Q1 were divided into the higher and lower AT groups, respectively. The Q1 and Q3 scores before the COVID-19 pandemic were 110 and 122, and were 112 and 124 during COVID-19, respectively. In total, 157 (average age = 22.56 years, $\mathrm{SD}=2.38$ ) and 139 individuals (average age $=$ 22.39 years, $\mathrm{SD}=2.55$ ) were categorized into the higher and lower ATs groups, respectively, before the COVID-19 pandemic; during the pandemic, 169 (average age $=22.47$ years, $\mathrm{SD}=3.40$ ) and 165 individuals (average age $=22.30$ years, $\mathrm{SD}=3.00$ ) were categorized into the higher and lower ATs groups, respectively. More detailed demographic information is listed in Tables 1 and 2.

\section{Measurements}

\section{Autism Spectrum Quotient (AQ)}

The Chinese version of AQ is a reliable instrument for quantifying ATs in both clinical and non-clinical samples in mainland China (Zhao et al., 2020). The continuous (four-point Likert) scale was used. In our study, the alpha for internal reliability (henceforth, $\alpha$ ) were .72 and .73 for the overall AQ collected before and during COVID-19, respectively.

\section{Emotional Experiences}

The PANAS is a 20 -item self-report measure of positive and negative emotions developed by Watson et al. (1988). The scale, as well as individual items or emotional words selected from the scale, has been used to measure the emotional impact of the COVID-19 pandemic and dynamic emotional changes during COVID-19 lockdown (CanetJuric et al., 2020; Huang et al., 2020; Moron \& BiolikMoron, 2021). The scale ranges from 1 (very slightly or not at all) to 5 (very much). In the present study, the time frame for responses regarding emotional experience was 'during the past two weeks'. The reliability of the positive emotions subscale obtained before and during COVID-19 were $\alpha=0.85$ and $\alpha=.88$, respectively; the reliability of the negative emotions subscale obtained before and during COVID-19 were $\alpha=.81$ and $\alpha=.90$, respectively.

We also used a demographic (Tables 1 and 2) and health survey. Participants' health was examined using the following questions:

1) Do you Have a Diagnosis of any Mental Health Condition? (E.G. Anxiety, Depression, and Schizophrenia)

2) Do you Have a Diagnosis of any Neurological Disease? (E.G. Epilepsy and Meningitis)

3) Do you Have a Diagnosis of any Serious Medical Illness? (E.G. Head Injury and Heart Disease)

4) Do you or your Relatives Have a Diagnosis of ASD?

5) Were you or your Relatives Confirmed as a COVID-19 Case? (this Question Was Only Asked during the Outbreak)

\section{Procedures}

\section{Before COVID-19}

The data were collected from September 17 to 27, 2019 during the freshmen's entrance education or class interval. Participants were informed about the voluntary nature of the study, guaranteed the anonymity and confidentiality of their data, and given a small gift (a ballpoint pen worth RMB 2.5) for participating. After the students gave their consent, we distributed the questionnaire. During data collection, the participants were asked to place the questionnaires in the corner of the table after they had completed the survey. The questionnaires were then collected on the spot.

\section{During COVID-19}

On January 20, 2020, the National Health Commission in China officially listed COVID-19 as a type B infectious disease, marking the actual beginning of the comprehensive 
Table 1 Background characteristics of the pre-COVID19 outbreak sample

\begin{tabular}{llll}
\hline higher ATs group $(n=157)$ & $\%(n)$ & lower ATs group $(n=139)$ & $\%(n)$ \\
\hline Gender & & Gender \\
Male & $52.23(82)$ & Male & $40.46(57)$ \\
Female & $47.78(75)$ & Female & $59.54(82)$ \\
Major & & Major & \\
Business administration & $19.11(30)$ & Business administration & $10.07(14)$ \\
pedagogy & $29.94(47)$ & pedagogy & $22.30(31)$ \\
psychology & $19.75(31)$ & psychology & $26.62(37)$ \\
engineering & $16.56(26)$ & engineering & $18.71(26)$ \\
biology & $14.65(23)$ & biology & $22.30(31)$ \\
\hline
\end{tabular}

upgrade of pandemic prevention and public concern. The data collection period was from February 6 to 16, 2020. Because the COVID-19 outbreak coincided with the Spring Festival, the students were on vacation, and the state called on everyone to be isolated at home; as such, offline data collection was rendered almost impossible. Therefore, the participants were asked to complete an online questionnaire hosted by Wenjuanxing (https://www.wjx.cn/). Previous studies have shown that there are differences in the level of autistic traits among students of different majors (Baron-Cohen et al., 2001) , to reduce the heterogeneity between participants before and during COVID-19, we only investigated college students with the same major before the outbreak. On the front page of the online questionnaire, we explained the purpose of this study in detail, and clarified the number of items in and the approximate time needed to complete the questionnaire. The participants were given RMB 2 for their participation.

\section{Statistical Analysis}

To exclude the false differences caused by possible measurement non-equivalence between the data before and during the pandemic, we conducted a multi-group confirmatory factor analysis (MCFA) in AMOS 20.0 to test the measurement
Table 2 Background characteristics of the duringCOVID-19 outbreak sample

\begin{tabular}{|c|c|c|c|}
\hline higher ATs group $(n=169)$ & $\%(n)$ & lower ATs group $(n=165)$ & $\%(n)$ \\
\hline Gender & & Gender & \\
\hline Male & $46.75(79)$ & Male & $46.67(77)$ \\
\hline Female & $53.25(90)$ & Female & $53.33(88)$ \\
\hline Major & & Major & \\
\hline Business administration & $20.12(34)$ & Business administration & $21.82(36)$ \\
\hline pedagogy & $23.67(40)$ & pedagogy & $19.39(32)$ \\
\hline psychology & $21.30(36)$ & psychology & $25.45(42)$ \\
\hline engineering & $21.89(37)$ & engineering & $18.18(30)$ \\
\hline biology & $13.02(22)$ & biology & $15.15(25)$ \\
\hline RL before outbreak & & RL before outbreak & \\
\hline Eastern China & $68.05(115)$ & Eastern China & $78.18(129)$ \\
\hline Central China & $13.61(23)$ & Central China & $11.52(19)$ \\
\hline Western China & $18.34(31)$ & Western China & $10.30(17)$ \\
\hline RL during outbreak & & RL during outbreak & \\
\hline Eastern China & $51.48(87)$ & Eastern China & $53.94(89)$ \\
\hline Central China & $19.53(33)$ & Central China & $16.36(27)$ \\
\hline Western China & $28.99(49)$ & Western China & $29.70(49)$ \\
\hline RA during outbreak & & RA during outbreak & \\
\hline Rural areas & $70.41(119)$ & Rural areas & $74.55(123)$ \\
\hline Urban areas & $29.59(50)$ & Urban areas & $25.45(42)$ \\
\hline Only child or not & & Only child or not & \\
\hline Yes & $36.09(61)$ & Yes & $51.52(85)$ \\
\hline Not & $63.91(108)$ & Not & $48.48(80)$ \\
\hline
\end{tabular}

Note: $\mathrm{RL}=$ Region of location; $\mathrm{RA}=$ Residence area 
invariance (Putnick \& Bornstein, 2016). Given that the AQ contains more items and dimensions, item parcelling strategy was used for MCFA to improve the index fit of the model (Rocha \& Chelladurai, 2012).

To confirm the suitability of analysis of variance (ANOVA) as the method for estimating the effect of categorical variables on emotions, we tested whether the dependent variables followed the normal distribution. The normal distribution test in SPSS showed all dependent variables following a non-normal distribution (negative emotions: $\mathrm{K}-\mathrm{S}=.085$, $\mathrm{df}=630, p<.05$, skewness $=.53$, kurtosis $=-.27$; fear: $\mathrm{K}-$ $\mathrm{S}=.171, \mathrm{df}=630, p<.05$, skewness $=.732$, kurtosis $=$ -.316 ; anger: $\mathrm{K}-\mathrm{S}=.241, \mathrm{df}=630, p<.05$, skewness $=$ 0.899 , kurtosis $=-.127$; positive emotions: $\mathrm{K}-\mathrm{S}=.059, \mathrm{df}=$ $630, p<.05$, skewness $=-.11$, kurtosis $=-.023)$. Therefore, we used a generalized linear model using maximum likelihood estimation to estimate the effect of independent variables (i.e. pandemic situation [before, during], gender [male, female], and ATs group [higher ATs group, lower ATs group]) on emotions.

\section{Results}

\section{Measurement Invariance Test}

The results of the MCFA are shown in Table 3. The fit indexes of these two unconstrained models were good and met the preconditions for subsequent equivalence testing. If the chisquared difference test of model comparison is not significant, then the comparison models can be considered equivalent in the corresponding test level (Meade et al., 2008). In the case of large samples, small chi-squared differences also indicate significant differences; when the chi-squared is indicated as significant, the difference of the fit index ( $\triangle \mathrm{CFI}$ and $\Delta \mathrm{TLI})$ is further used to detect equivalence - that is, when $\triangle \mathrm{CFI}$ and $\Delta$ TLI are both less than .01 (Meade et al., 2008) - then the comparison models do not have a significant difference. The results of the model comparison showed no significant difference between the fit indexes of the baseline model and the model with equal defining factor loading (Model A vs. Model B), and between the fit indexes of Model B and the model with equal defining factor loading and covariances (Model B vs. Model C) for both AQ and PANAS. Thus, the null hypothesis of invariance should not be rejected under moderate loose criterion (Wasti et al., 2007). In other words, the understanding of the same construct and the true score of potential factors among the two groups measured at different time points were consistent, thereby meeting the conditions for the subsequent comparison between the time points of before and during the COVID-19 pandemic.

\section{Effects of COVID-19, Gender, and Autistic Traits on Emotions}

The detailed descriptive statistics are shown in Table 4.

The results of the statistical test are shown in Table 5.

In the analysis of positive emotions, we found that the higher ATs group experienced significantly fewer positive emotions compared with the lower ATs group $(95 \%$ Wald CI for difference: $-4.496,-2.500)$. The level of positive emotions during COVID-19 was significantly lower than that before COVID-19 (95\% Wald CI for difference: -4.090 , $-2.093)$.

Regarding negative emotions, the level of negative emotions during COVID-19 was significantly lower than that before COVID-19 (95\% Wald CI for difference: -4.090 , $-2.093)$. The level of negative emotions experienced by the

Table 3 Fit indexes of model equivalence and results of the nested model comparison

\begin{tabular}{|c|c|c|c|c|c|c|c|c|c|c|c|}
\hline Model & $x^{2}$ & $d f$ & CFI & TLI & RMSEA & Model-C & $\Delta \times 2$ & $\Delta d f$ & $p$ & $\Delta \mathrm{CFI}$ & $\Delta \mathrm{TLI}$ \\
\hline \multicolumn{12}{|c|}{ PANAS } \\
\hline A & 1189.686 & 324 & .918 & .904 & .047 & & & & & & \\
\hline $\mathrm{B}$ & 1246.594 & 342 & .914 & .905 & .047 & (1) & 56.908 & 18 & $<.01$ & -.002 & .001 \\
\hline $\mathrm{C}$ & 1250.646 & 345 & .914 & .906 & .047 & (2) & 4.052 & 3 & $>.05$ & .000 & .001 \\
\hline $\mathrm{D}$ & 1443.983 & 372 & .899 & .896 & .049 & (3) & 193.336 & 27 & $<.01$ & -.015 & .009 \\
\hline \multicolumn{12}{|l|}{ AQ } \\
\hline A & 36.607 & 8 & .962 & .904 & .054 & & & & & & \\
\hline B & 39.531 & 12 & .963 & .939 & .044 & (1) & 2.924 & 4 & $>.05$ & .001 & .035 \\
\hline $\mathrm{C}$ & 40.321 & 13 & .963 & .944 & .042 & (2) & .790 & 1 & $>.05$ & .000 & .005 \\
\hline $\mathrm{D}$ & 74.182 & 18 & .925 & .916 & .051 & (3) & 33.861 & 5 & $<.01$ & -.038 & -.028 \\
\hline
\end{tabular}

Note: $\mathrm{A}=$ unconstrained baseline model, $\mathrm{B}=$ measurement weights, $\mathrm{C}=$ structural covariances, $\mathrm{D}=$ measurement residuals; the grey shaded part shows the comparison results, Model C = model comparison, (1) = Model A vs. Model B, (2)= Model B vs. Model C, (3) = Model C vs. Model D 
Table 4 Descriptive statistics on variables of interest

\begin{tabular}{|c|c|c|c|c|c|c|c|c|c|}
\hline & & \multicolumn{4}{|l|}{ Male } & \multicolumn{4}{|l|}{ Female } \\
\hline & & \multicolumn{2}{|l|}{ L-ATs } & \multicolumn{2}{|l|}{ H-ATs } & \multicolumn{2}{|l|}{ L-ATs } & \multicolumn{2}{|l|}{ H-ATs } \\
\hline & & $\begin{array}{l}\text { Before } \\
(n=57)\end{array}$ & $\begin{array}{l}\text { During } \\
(n=77)\end{array}$ & $\begin{array}{l}\text { Before } \\
(n=82)\end{array}$ & $\begin{array}{l}\text { During } \\
(\mathrm{n}=79)\end{array}$ & $\begin{array}{l}\text { Before } \\
(n=82)\end{array}$ & $\begin{array}{l}\text { During } \\
(n=88)\end{array}$ & $\begin{array}{l}\text { Before } \\
(n=75)\end{array}$ & $\begin{array}{l}\text { During } \\
(n=90)\end{array}$ \\
\hline \multirow[t]{3}{*}{ AQ } & M & 102.44 & 104.74 & 127.57 & 129.85 & 101.63 & 105.26 & 126.76 & 129.56 \\
\hline & SD & $(7.26)$ & $(6.20)$ & $(4.30)$ & $(5.47)$ & $(6.20)$ & $(5.40)$ & $(3.87)$ & $(4.48)$ \\
\hline & Range & [76-109] & [81-111] & [123-143] & {$[125-147]$} & [84-109] & {$[85-111]$} & [123-143] & [125-152] \\
\hline \multirow[t]{3}{*}{ Positive emotions } & M & 32.07 & 29.75 & 28.79 & 26.18 & 32.83 & 27.70 & 27.85 & 25.54 \\
\hline & SD & $(6.56)$ & $(7.65)$ & $(6.44)$ & $(7.18)$ & $(6.30)$ & $(6.41)$ & $(5.47)$ & $(5.19)$ \\
\hline & Range & [19-46] & {$[12-48]$} & {$[11-41]$} & {$[10-46]$} & {$[14-47]$} & [12-38] & [14-38] & [11-37] \\
\hline \multirow[t]{3}{*}{ Negative emotions } & M & 20.16 & 16.29 & 25.62 & 22.36 & 18.90 & 19.25 & 23.11 & 24.76 \\
\hline & $\mathrm{SD}$ & $(6.39)$ & $(5.68)$ & $(6.76)$ & $(7.18)$ & $(5.70)$ & $(6.78)$ & $(6.54)$ & $(6.55)$ \\
\hline & Range & {$[11-41]$} & {$[10-32]$} & {$[12-45]$} & {$[10-42]$} & {$[10-46]$} & [10-39] & {$[10-39]$} & [11-39] \\
\hline \multirow[t]{3}{*}{ Scared and Afraid } & M & 3.32 & 3.11 & 4.65 & 4.44 & 3.24 & 4.15 & 4.61 & 5.26 \\
\hline & 'SD & $(1.57)$ & $(1.56)$ & (1.94) & $(2.09)$ & $(1.59)$ & (1.79) & $(2.16)$ & $(1.75)$ \\
\hline & Range & {$[2-8]$} & {$[2-8]$} & {$[2-10]$} & {$[2-10]$} & [2-10] & [2-9] & [2-9] & [2-9] \\
\hline \multirow[t]{3}{*}{ Irritable } & M & 1.79 & 1.65 & 2.09 & 2.37 & 1.77 & 1.81 & 1.88 & 2.61 \\
\hline & SD & $(.99)$ & $(.86)$ & (.98) & (1.14) & $(.93)$ & $(.97)$ & $(.96)$ & $(1.02)$ \\
\hline & Range & {$[1-5]$} & {$[1-4]$} & {$[1-5]$} & {$[1-5]$} & {$[1-5]$} & {$[1-5]$} & {$[1-4]$} & {$[1-5]$} \\
\hline
\end{tabular}

higher ATs group was significantly higher as compared to that reported by lower ATs group (95\% Wald CI for difference: $4.300,6.328$ ). We also observed a significant interaction effect between pandemic situation and gender, as shown in fig. 1a. Further analysis indicated that the level of negative emotions during COVID-19 was lower than that before COVID-19 in males (95\% Wald CI for difference: -5.047 , $-2.080 ; p<.05)$, but this difference was not present among females (95\% Wald CI for difference: $-.383,2.380)$.

The level of anger experienced by the higher ATs group was significantly higher than that of the lower ATs group (95\% Wald CI for difference: .328, .636), whereas the level of anger during the COVID-19 pandemic was significantly higher than that before (95\% Wald CI for difference: .073, .382). We found a significant interaction effect between pandemic situation and gender, as shown in fig. 1b. Further analysis indicated that anger level during the pandemic was higher than that before in females (95\% Wald CI for difference: .174, .595 ; $p<0.05)$, but not among males $(95 \%$ Wald CI for difference: $-.155, .297)$. We also found a significant interaction effect between ATs group and pandemic situation, as shown in fig. 1c. Further analysis indicated that anger during the pandemic was of a higher level than that before in the higher ATs group (95\% Wald CI for difference: .293, .720; $p<.05$ );
Table 5 Statistical test results on the effect of independent variables on emotions

\begin{tabular}{lllllllll}
\hline \multirow{2}{*}{ source } & \multicolumn{7}{l}{ dependent variable- emotions } \\
\cline { 2 - 9 } & Anger & & Fear & & positive emotions & \multicolumn{2}{c}{ negative emotions } \\
\hline & Wald $\chi^{2}$ & Sig. $(p)$ & Wald $\chi^{2}$ & Sig. $(p)$ & Wald $\chi^{2}$ & Sig. $(p)$ & Wald $\chi^{2}$ & Sig. $(p)$ \\
Gender & .308 & .579 & 8.864 & .003 & 1.972 & .160 & .585 & .444 \\
ATs group & 37.479 & .000 & 77.306 & .000 & 47.136 & .000 & 105.558 & .000 \\
COVID-19 & 8.358 & .004 & 3.837 & .050 & 36.830 & .000 & 6.150 & .013 \\
Interaction 1 & 12.501 & .000 & .208 & .649 & 1.526 & .217 & .860 & .354 \\
Interaction 2 & 3.97 & .046 & 11.133 & .001 & 1.507 & .220 & 19.448 & .000 \\
Interaction 3 & .096 & .757 & .094 & .759 & .019 & .890 & .787 & .375 \\
Interaction 4 & .738 & .390 & .194 & .660 & 2.336 & .126 & .109 & .741 \\
\hline
\end{tabular}

Note: Interaction $1=$ ATs group $*$ COVID-19; Interaction $2=$ gender $*$ COVID-19; Interaction $3=$ gender $*$ ATs group; Interaction 4 = gender * ATs group* COVID-19; $\mathrm{df}=1$ for all models 
Fig. 1 Gender $\times$ COVID-19 situation interaction and ATs group $\times$ COVID-19 situation interaction with respect to negative emotions, anger and fear
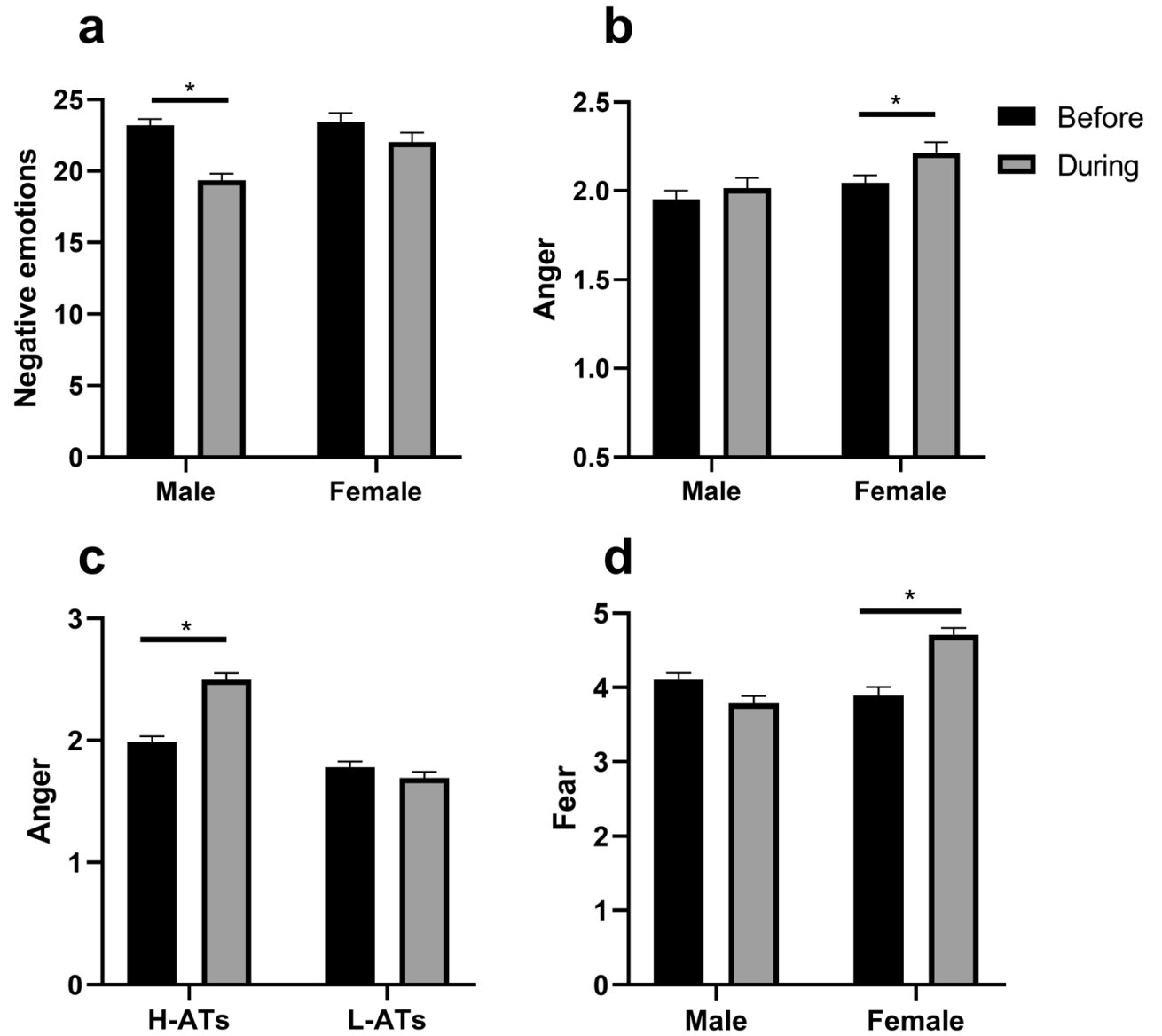

Female this difference was not present in the lower ATs group (95\% Wald CI for difference: $-.274, .173$ ).

The level of fear experienced by females was significantly higher than that of males (95\% Wald CI for difference: .149, .721). The level of fear during the COVID-19 pandemic was marginally significantly higher than that before $(95 \%$ Wald CI for Difference: .000, .572). The level of fear experienced by the higher ATs group was significantly higher compared with the lower ATs group (95\% Wald CI for difference: .997, 1.570). The results also indicated a significant interaction effect between pandemic situation and gender, as shown in fig. $1 \mathrm{~d}$ : fear during the COVID-19 pandemic was higher than that before in females (95\% Wald CI for difference: .383, 1.163; $p<.05$ ) but not in men (95\% Wald CI for difference: -.620 , $.218)$.

\section{Discussion}

The present study aimed to examine the emotional impact of the COVID-19 pandemic as well as its moderating effects of gender and ATs. Our analysis revealed that the COVID-19 pandemic reduced positive emotions and increased fear and anger. More importantly, individuals with higher ATs and females were found more susceptible to emotional changes before and during the COVID-19 pandemic. The strengths of this research include the comparison of emotions before and during the COVID-19 pandemic, large sample with a relatively balanced gender ratio, and use of ATs as a moderator. The core contribution to the body of knowledge is the extension of the association between ASD and enhanced stress response to the COVID-19 pandemic.

Consistent with the results of a recent psycholinguistic analysis ( $\mathrm{Li}$ et al., 2020), our findings showed that people's positive emotions significantly decreased during the COVID19 pandemic, suggesting that the levels of enthusiasm, vitality, and immense interest during the pandemic were lower than those before. In the face of a potential disease threat like COVID-19, a set of adaptive psychological mechanisms, namely, the behavioural immune system (BIS), is activated to trigger avoidance behaviour or motivation for self-protection, leading people to act in a more reticent and conservative manner (Li et al., 2020; Olivera-La Rosa et al., 2020). Meanwhile, as the main dimension of human emotional experiences, as measured by the PANAS, positive emotions reflect the activation of the behavioural activation system (BAS), which contributes to approach-related behaviour and motivation (Schiltz et al., 2018; Zhao et al., 2020). Thus, BAS and 
BIS have a certain degree of functional compatibility. Therefore, we suggested that the decreased positive emotions during the COVID-19 pandemic may partly reflect the adaptive decrease of BAS that is caused by BIS.

Contrary to our research hypothesis, our results showed that the overall negative emotion during COVID-19 was lower than that before. However, this effect was only found in males. Gender differences in attitudes and behaviours to the ongoing COVID-19 situation may contribute to this seemingly counterintuitive phenomenon. The COVID-19 pandemic has not always had negative consequences. The extensive and effective anti-pandemic action in China, such as the sending of aid in terms of materials and medical personnel from provinces across the country to Wuhan, had a broad and positive social impact, such as increased collective cohesion and patriotism ( $\mathrm{Li}$ et al., 2020). Males have tended to be more concerned with the social impact of COVID-19 than females (Isabelle \& Kleinberg, 2020). Therefore, we suggested that men would be more likely to be permeated by these positive effects, diluting their overall negative emotions, compared with women. In addition, males have been reported to take fewer family and care responsibilities than females during the pandemic (Etheridge \& Spantig, 2020). Meanwhile, the data for during COVID-19 was collected during the Spring Festival only. Men are more likely than women to enjoy leisure time on holidays (Moron \& Biolik-Moron, 2021), which may explain their lower overall negative emotions in our study. However, these are speculations, and the role of other variables in this gender difference, such as coping strategies and emotional regulation during the COVID-19 pandemic, needs to be further examined.

The discrete negative emotions approach is more informative for describing the emotional impacts of the COVID-19 pandemic compared with the overall measurement of negative emotions (Moron \& Biolik-Moron, 2021). Consistent with recent studies ( $\mathrm{Li}$ et al., 2020; Moron \& Biolik-Moron, 2021; Wang et al., 2021), our results showed that the levels of fear and anger during the COVID-19 pandemic were significantly higher than those before. However, it should be noted that the enhanced fear and anger during the pandemic are likely derived from different sources. Specifically, fear is the core emotional response to an impending risk of viral infection (Moron \& Biolik-Moron, 2021; Schimmenti et al., 2020). Although fear is an undesirable emotional experience, as indicated by the theories of BIS and perceived risk ( $\mathrm{Li}$ et al., 2020; Makhanova \& Shepherd, 2020), fear-related emotions can prompt individuals faced with a potential pathogen threat to discover potential infection sources in time and take action to avoid infection. The increased anger during the pandemic seems to reflect the negative effects associated with anti-pandemic measures, such as lockdown and self-isolation at home (Brooks et al., 2020;
Li et al., 2020; Moron \& Biolik-Moron, 2021). Therefore, the authorities must also seek to minimize the collateral negative effects of public health initiatives.

Our results further revealed that the changes in anger and fear before and during the COVID-19 pandemic were more prominent among females, which is in line with recent findings on females reporting high levels of fear and anger than males during the COVID-19 pandemic (Huang et al., 2020; van der Vegt \& Kleinberg, 2020). Recent studies have shown that females are more concerned with disease infection risk than males. For example, females perceive COVID-19 as more prevalent and lethal than males do (Galasso et al., 2020; Isabelle \& Kleinberg, 2020; Oreffice \& QuintanaDomeque, 2021). Based on the robust association between perceived risk and increased fear (Zheng et al., 2019), we held that the enhanced risk perception of being infected in females would likely increase their fear. Studies measuring fear and risk perception are needed to validate this hypothesis. In terms of the increased anger among females, their lower tolerance for the inconsistent dissemination of information on the transmission of COVID-19 from person to person ( $\mathrm{Li}$ et al., 2020) as well as the increased burden of family care and job risk caused by the COVID-19 outbreak among females (Barrett, 2020; Etheridge \& Spantig, 2020) are likely to increase their anger. In addition, the COVID-19 pandemic forces people to face more uncertainties (Zandifar \& Badrfam, 2020), while higher intolerance of uncertainty is associated with enhanced negative emotions (e.g. anxiety and anger) during COVID-19 pandemic (Hamama-Raz et al., 2021; Valle et al., 2020). A recent study has shown that females have higher levels of intolerance of uncertainty than males (Valle et al., 2020). Therefore, the gender differences in intolerance of uncertainty may partly contribute to the gender differential susceptibility to the emotional impact of COVID-19 pandemic.

Independent of the COVID-19 pandemic condition, our results showed that individuals with higher ATs reported fewer positive emotions and more negative emotions compared with individuals with lower ATs. These findings are consistent with previous studies: emotional experiences as measured by the PANAS in individuals with ASD are more negative and less positive compared with TD controls (Zhao et al., 2020), supporting the similarity hypothesis of ATs in the general and clinical ASD population (Baron-Cohen et al., 2001).

Scholars have hypothesized on an intrinsic relation between impaired stress mechanisms and autism spectrum (Haruvi-Lamdan et al., 2020; Haruvi-Lamdan et al., 2019; van Oort et al., 2020). However, the evidence is scarce on whether individuals with ASD also show increased stress response to COVID-19 pandemic, which affects the entire population at the same time. Through an analogy-ASD approach that grouped the general population into higher and lower ATs segments, our study showed that individuals with higher ATs were more susceptible to emotional changes before and during 
the COVID-19 pandemic than those with lower ATs, as reflected in the significantly higher levels of anger experienced during COVID-19 than those before. To some extent, this result is consistent with Valenti et al.'s observation (Valenti et al., 2012) that individuals with ASD shown to have lower adaptive function as compared to that in TD controls after an earthquake.

There are two possible explanations for the higher level of anger experienced by individuals with higher ATs during the COVID-19 pandemic. Firstly, the inconsistent dissemination of information by the relevant responsible units in Hubei Province on whether COVID-19 was transmitted from person to person led to moral outrage (Li et al., 2020). Previous studies have shown that moral judgment in individuals with ASD is more rigorous (Margoni \& Surian, 2016). Therefore, individuals with higher ATs may have judged the spread of misinformation as morally more impermissible cosmpared with those with lower ATs, resulting in an increased indignation in the former. Secondly, the COVID-19 pandemic exposed the public to unprecedented uncertainty (Valle et al., 2020), and the anti-pandemic measures have tended to force the public to make rapid changes to their daily habits, established plans, and even social contacts (Brooks et al., 2020; Moron \& Biolik-Moron, 2021). ASDs or ATs are associated with a lower tolerance for uncertainty and unexpected change (Cassidy et al., 2020; Hirvikoski \& Blomqvist, 2015) and with impaired executive function (Zhao et al., 2019), which may make it difficult for individuals with higher ATs to manage effectively the series of changes caused by the COVID-19 pandemic and lead to them becoming more irritable. Finally, atypical emotional regulation and poor social support as a result of difficulties in social communication (Cassidy et al., 2020; Zhao et al., 2020) may further increase the levels of anger experienced by individuals with higher ATs.

Although our hypothesis is consistent with some theoretical and empirical studies (Masi et al., 2015; Saresella et al., 2009; Tanu \& Kakkar, 2019; Zhao et al., 2021) that compared with individuals with lower ATs, the fear level experienced by individuals with higher ATs is significantly higher than that before the pandemic, our results did not support this inference. Experimental designs (e.g. situational priming of pathogen threat) could be helpful to understand the relationship between ATs and fear response to the COVID-19 pandemic.

Our findings suggested improving emotions of females and individuals with higher ATs during the COVID-19 pandemic should be given priority. The development of consistent policies and procedures to ensure the timely update of information related to COVID-19 would help reduce the perceived lack of control and certainty, which would mitigate the fear experienced by females. Improving scientific bases and effectiveness of anti-pandemic actions, and providing new action plans for individuals with higher ATs would help reduce the anger felt by these groups. Finally, improving psychological anti-pandemic capability, such as emotion regulation skills, is also helpful to ameliorate the negative emotions that caused by COVID-19.

The present study had the following limitations. Firstly, different samples were evaluated before and during the COVID-19 pandemic, although the use of the same major categories and large sample size could effectively eliminate the heterogeneity between the groups. Nonetheless, the extent of the homogeneity of the two groups and of the reliability of the pre-pandemic measurements as a baseline in highlighting the emotional impact of the COVID-19 pandemic remain unclear. In addition, the data collection was only during the Spring Festival, which could have potentially interfered with the emotional impact of the COVID19 pandemic. These inherent limitations of the transversal design did not allow for causal association to be established (test-retest) (González-Valero et al., 2020), and as such, the findings obtained ought to be interpreted with caution. Secondly, the sample in this study was only composed of college students. The moderating effect of ATs on the emotional impacts of the COVID-19 pandemic could differ in autistic populations, which warrant further investigation. Thirdly, the self-report method may not be effective in ensuring health screening, and individuals with preexisting mental disorders may be at higher risk of relapse or new episodes of their disorder during the COVID-19 pandemic (Rajkumar, 2020). Therefore, our results may be potentially affected by the data of the participants missed by the health screening. Lastly, the emotional experience during the pandemic changes dynamically over time (Canet-Juric et al., 2020), and our findings only reflected the early stage of the pandemic development. Emotional impact and its regulatory factors in the later stage of the pandemic development need to be further explored.

\section{Conclusions}

The present study revealed that the COVID-19 outbreak reduced the public's positive emotions and increased fear and anger. More importantly, females and individuals with higher ATs showed increased susceptibility to pre- and duringpandemic emotion changes. These findings have implications for pandemic crisis interventions. Meanwhile, our preliminary results provide interesting bases for further examination in future studies.

Data Availability The data-sets are available from the corresponding author on reasonable request.

\section{Declarations}

Ethics Approval All participants were informed about the study and provided written or electronic informed consent before the study. 
Conflict of Interests The authors declare that they have no conflicts of interests.

\section{References}

Baron-Cohen, S., Wheelwright, S., Skinner, R., Martin, J., \& Clubley, E. (2001). The autism-Spectrum quotient (AQ): Evidence from Asperger syndrome/high-functioning autism, Malesand females, Scientists and Mathematicians. Journal of autism and developmental disorders. 31(1), 5-17. https://doi.org/10.1023/A:1005653411471

Barrett, D. (2020). Dreams about COVID-19 versus normative dreams: Trends by gender. Dreaming, 30(3), 216-221. https://doi.org/10. 1037/drm0000149

Bishop-Fitzpatrick, L., Mazefsky, C. A., Minshew, N. J., \& Eack, S. M. (2015). The relationship between stress and social functioning in adults with autism Spectrum disorder and without intellectual disability. Autism Research, 8(2), 164-173. https://doi.org/10.1002/ aur.1433

Bishop-Fitzpatrick, L., Minshew, N. J., Mazefsky, C. A., \& Eack, S. M. (2017). Perception of life as stressful, not biological response to stress, is associated with greater social disability in adults with autism Spectrum disorder. Journal of Autism and Developmental Disorders, 47(1), 1-16. https://doi.org/10.1007/s10803-016-2910-6

Brooks, S. K., Webster, R. K., Smith, L. E., Woodland, L., Wessely, S., Greenberg, N., \& Rubin, G. J. (2020). The psychological impact of quarantine and how to reduce it: Rapid review of the evidence. The Lancet, 395(10227), 912-920. https://doi.org/10.1016/s01406736(20)30460-8

Canet-Juric, L., Andrés, M. L., del Valle, M., López-Morales, H., Poó, F., Galli, J. I., Yerro, M., \& Urquijo, S. (2020). A longitudinal study on the emotional impact cause by the COVID-19 pandemic quarantine on general population. Frontiers in Psychology, 11, 2431. https:// doi.org/10.3389/fpsyg.2020.565688

Cassidy, S. A., Nicolaidis, C., Davies, B., Rosa, S. D. R., Eisenman, D., Onaiwu, M. G., Kapp, S. K., Kripke, C. C., Rodgers, J., \& Waisman, T. C. (2020). An expert discussion on autism in the COVID-19 pandemic. Autism in Adulthood, 2(2), 106-117. https://doi.org/10.1089/aut.2020.29013.sjc

Etheridge, B., \& Spantig, L. (2020). The gender gap in mental well-being during the Covid-19 outbreak: Evidence from the UK (No. 202008). Institute for Social and Economic Research, 2020-08. Institute for Social and Economic Research

Farhood, L., Fares, S., \& Hamady, C. (2018). Correction to: PTSD and gender: Could gender differences in war trauma types, symptom clusters and risk factors predict gender differences in PTSD prevalence? Archives of Women's Mental Health, 21(6), 735-743. https:// doi.org/10.1007/s00737-018-0874-6

Galasso, V., Pons, V., Profeta, P., Becher, M., Brouard, S., \& Foucault, M. (2020). Gender differences in Covid-19 related attitudes and behavior: Evidence from a panel survey in eight OECD countries. Social ence Electronic Publishing.

González-Valero, G., Zurita-Ortega, F., Lindell-Postigo, D., Conde-Pipó, J., Grosz, W. R., \& Badicu, G. (2020). Analysis of self-concept in adolescents before and during COVID-19 lockdown: Differences by gender and sports activity. Sustainability, 12(18), 7792. https://doi. org $/ 10.3390 /$ su 12187792

Hamama-Raz, Y., Goodwin, R., Leshem, E., \& Ben-Ezra, M. (2021). Intolerance of uncertainty and mental health during the covid-19 pandemic: The role of anger as a moderator. Journal of Psychiatric Research, 138(3), 50-52.

Haruvi-Lamdan, N., Horesh, D., Zohar, S., Kraus, M., \& Golan, O. (2020). Autism Spectrum Disorder and Post-Traumatic Stress Disorder: An unexplored co-occurrence of conditions. Autism : the international journal of research and practice, 24(4), 884-898. https://doi.org/10.1362361320912143/1362361320912143

Haruvi-Lamdan, N., Lebendiger, S., Golan, O., \& Horesh, D. (2019). Are PTSD and autistic traits related? An examination among typically developing Israeli adults. Comprehensive Psychiatry, 89, 22-27. https://doi.org/10.1016/j.comppsych.2018.11.004

Hirvikoski, T., \& Blomqvist, M. (2015). High self-perceived stress and poor coping in intellectually able adults with autism spectrum disorder. Autism, 19(6), 752-757. https://doi.org/10.1177/ 1362361314543530

Huang, L., Lei, W., Xu, F., Liu, H., \& Yu, L. (2020). Emotional responses and coping strategies in nurses and nursing students during Covid19 outbreak: A comparative study. PLoS One, 15(8), e 0237303. https://doi.org/10.1371/journal.pone.0237303

Isabelle, V. D. V., \& Kleinberg, B. (2020). Women worry about family, men about the economy: Gender differences in emotional responses to COVID-19. International Conference on Social Informatics, 397-409. https://doi.org/10.1007/978-3-030-60975-7_29

Li, S., Wang, Y., Xue, J., Zhao, N., \& Zhu, T. (2020). The impact of COVID-19 epidemic declaration on psychological consequences: A study on active Weibo users. International Journal of Environmental Research and Public Health, 17(6), 2032. https:// doi.org/10.3390/ijerph17062032

Kildahl, A. N., Bakken, T. L., Iversen, T. E., \& Helverschou, S. B. (2019). Identification of post-traumatic stress disorder in individuals with autism Spectrum disorder and intellectual disability: A systematic review. Journal of Mental Health Research in Intellectual Disabilities, 12(1-2), 1-25. https://doi.org/10.1080/19315864. 2019.1595233

Makhanova, A., \& Shepherd, M. A. (2020). Behavioral immune system linked to responses to the threat of COVID-19. Pers Individ Dif, 167, 110221. https://doi.org/10.1016/j.paid.2020.110221

Margoni, F., \& Surian, L. (2016). Mental state understanding and moral judgment in children with autistic Spectrum disorder. Frontiers in Psychology, 7, 1478. https://doi.org/10.3389/fpsyg.2016.01478

Masi, A., Quintana, D. S., Glozier, N., Lloyd, A. R., Hickie, I. B., \& Guastella, A. J. (2015). Cytokine aberrations in autism spectrum disorder: A systematic review and meta-analysis. Molecular Psychiatry, 20(4), 440-446. https://doi.org/10.1038/mp.2014.59

Meade, A. W., Johnson, E. C., \& Braddy, P. W. (2008). Power and sensitivity of alternative fit indices in tests of measurement invariance. Journal of Applied Psychology, 93(3), 568-592.

Moron, M., \& Biolik-Moron, M. (2021). Trait emotional intelligence and emotional experiences during the COVID-19 pandemic outbreak in Poland: A daily diary study. Pers Individ Dif, 168, 110348. https:// doi.org/10.1016/j.paid.2020.110348

Olivera-La Rosa, A., Chuquichambi, E. G., \& Ingram, G. P. D. (2020). Keep your (social) distance: Pathogen concerns and social perception in the time of COVID-19. Pers Individ Dif, 166, 110200. https:// doi.org/10.1016/j.paid.2020.110200

Oreffice, S., \& Quintana-Domeque, C. (2021). Gender inequality in COVID-19 times: Evidence from UK prolific participants. Journal of Demographic Economics, 87(2), 261-287. https://doi.org/10. 1017/dem.2021.2

Putnick, D. L., \& Bornstein, M. H. (2016). Measurement invariance conventions and reporting: The state of the art and future directions for psychological research. Developmental Review, 41, 71-90. https://doi.org/10.1016/j.dr.2016.06.004

Rajkumar, R. P. (2020). COVID-19 and mental health: A review of the existing literature. Asian Journal of Psychiatry, 52, 102066. https:// doi.org/10.1016/j.ajp.2020.102066

Rocha, C. M., \& Chelladurai, P. (2012). Item parcels in structural equation modeling: An applied study in sport management. International Journal of Psychology \& Behavioral Sciences, 2(1), 46-53.

Saresella, M., Marventano, I., Guerini, F. R., Mancuso, R., Ceresa, L., Zanzottera, M., Rusconi, B., Maggioni, E., Tinelli, C., \& Clerici, M. 
(2009). An autistic endophenotype results in complex immune dysfunction in healthy siblings of autistic children. Biological Psychiatry, 66(10), 978-984. https://doi.org/10.1016/j.biopsych. 2009.06.020

Schiltz, H. K., McVey, A. J., Barrington, A., Haendel, A. D., Dolan, B. K., Willar, K. S., et al. (2018). Behavioral inhibition and activation as a modifier process in autism spectrum disorder: Examination of self-reported BIS/BAS and alpha EEG asymmetry. Autism Research, 11(12), 1653-1666. https://doi.org/10.1002/aur.2016

Schimmenti, A., Billieux, J., \& Starcevic, V. (2020). The four horsemen of fear: An integrated model of understanding fear experiences during the COVID-19 pandemic. Clinical Neuropsychiatry, 17(2), 4145. https://doi.org/10.36131/CN20200202

Shavit, T., Shahrabani, S., Benzion, U., \& Rosenboim, M. (2013). The effect of a forest fire disaster on emotions and perceptions of risk: A field study after the Carmel fire. Journal of Environmental Psychology, 36, 129-135. https://doi.org/10.1016/j.jenvp.2013.07. 018

Spratt, E. G., Nicholas, J. S., Brady, K. T., Carpenter, L. A., Hatcher, C. R., Meekins, K. A., Furlanetto, R. W., \& Charles, J. M. (2012). Enhanced cortisol response to stress in children in autism. Journal of Autism and Developmental Disorders, 42(1), 75-81. https://doi. org/10.1007/s10803-011-1214-0

Tanu, \& Kakkar, D. (2019). Influence of emotional imagery on risk perception and decision making in autism Spectrum disorder. Neurophysiology, 51(4), 281-292. https://doi.org/10.1007/s11062019-09822-8

Taylor, J. L., \& Corbett, B. A. (2014). A review of rhythm and responsiveness of cortisol in individuals with autism spectrum disorders. Psychoneuroendocrinology, 49, 207-228. https://doi.org/10.1016/j. psyneuen.2014.07.015

Valenti, M., Ciprietti, T., Egidio, C. D., Gabrielli, M., Masedu, F., Tomassini, A. R., \& Sorge, G. (2012). Adaptive response of children and adolescents with autism to the 2009 earthquake in L'Aquila, Italy. Journal of Autism and Developmental Disorders, 42(6), 954-960. https://doi.org/10.1007/s10803-011-1323-9

Valle, M., Andrés, M. L., Urquijo, S., Yerro, M., \& Juric, L. C. (2020). Intolerance of uncertainty over covid-19 pandemic and its effect on anxiety and depressive symptoms. Revista Interamericana de psicologia/Interamerican Journal of Psychology, 54(2), e1335.

van der Vegt, I., \& Kleinberg, B. (2020). Women worry about family, men about the economy: Gender differences in emotional responses to COVID-19. In International Conference on Social Informatics (pp. 397-409). Springer van Oort, J., Kohn, N., Vrijsen, J. N., Collard, R., Duyser, F. A., Brolsma, S. C. A., Fernández, G., Schene, A. H., Tendolkar, I., \& van Eijndhoven, P. F. (2020). Absence of default mode downregulation in response to a mild psychological stressor marks stressvulnerability across diverse psychiatric disorders. Neuroimage Clin, 25, 102176. https://doi.org/10.1016/j.nicl.2020.102176

Wang, J., Wang, Z., Liu, X., Yang, X., Zheng, M., \& Bai, X. (2021). The impacts of a COVID-19 epidemic focus and general belief in a just world on individual emotions. Personality and Individual Differences, 168, 110349. https://doi.org/10.1016/j.paid.2020. 110349

Wasti, S. A., Tan, H. H., Brower, H. H., \& Önder, Ç. (2007). Crosscultural measurement of supervisor trustworthiness: An assessment of measurement invariance across three cultures. Leadership Quarterly, 18(5), 477-489.

Watson, D., Clark, L. A., \& Tellegen, A. (1988). Development and validation of brief measures of positive and negative affect: The PANAS scales. Journal of Personality and Social Psychology, 54(6), 1063-1070.

Zandifar, A., \& Badrfam, R. (2020). Iranian mental health during the covid-19 epidemic. Asian Journal of Psychiatry, 51, 101990.

Zhao, X., Shi, W., Li, X., Li, W., \& Li, C. (2021). Autistic traits and negative emotions in the general population during covid-19: Mediating roles of the behavioural immune system and covid-19 risk perception. Psychiatry Research, 300(2), 113918.

Zhao, X., Li, X., Song, Y., Li, C., \& Shi, W. (2020). Autistic traits and emotional experiences in Chinese college students: Mediating role of emotional regulation and sex differences. Research in Autism Spectrum Disorders, 77, 101607. https://doi.org/10.1016/j.rasd. 2020.101607

Zhao, X., Li, X., Song, Y., \& Shi, W. (2019). Autistic traits and prosocial behaviour in the general population: Test of the mediating effects of trait empathy and state empathic concern. Journal of Autism and Developmental Disorders, 49(10), 3925-3938. https://doi.org/10. 1007/s10803-018-3745-0

Zheng, C., Zhang, J., Guo, Y., Zhang, Y., \& Qian, L. (2019). Disruption and reestablishment of place attachment after large-scale disasters: The role of perceived risk, negative emotions, and coping. International Journal of Disaster Risk Reduction, 40, 101273. https://doi.org/10.1016/j.ijdrr.2019.101273

Publisher's Note Springer Nature remains neutral with regard to jurisdictional claims in published maps and institutional affiliations. 